\title{
Study on Real-time Adjustment of Train Operation under the Single Direction Blockade of Existing Busy Trunk Lines Condition
}

\author{
Zhi-Zhong HUANG ${ }^{1,2, a}$, Jie XU ${ }^{1, b,}$, Shi-Lin LI ${ }^{1,2, c}$ and Li-Min JIA ${ }^{1, d}$ \\ ${ }^{1}$ State Key Laboratory of Rail Traffic Control and Safety, Beijing Jiaotong University, Beijing 100044, \\ China \\ ${ }^{2}$ School of Traffic and Transportation, Beijing Jiaotong University, Beijing 100044, China \\ azzhuang23@bjtu.edu.cn, bxujie@m.bjtu.edu.cn, ${ }^{\text {c} 15120838 @ b j t u . e d u . c n, ~ I I m j i a @ b j t u . e d u . c n ~}$ \\ ${ }^{*}$ Corresponding author
}

Keywords: Existing busy main lines, Interval blockade, Real-time adjustment, Linear programming.

\begin{abstract}
It is one of the common problems in the daily transportation organization of railway because of various disturbances that cause the train to deviate from the original operation plan. In this paper, the objective function is the minimum weighted summation of the train cancellation penalty value and the train late penalty value, classify each train according to the different speed of transportation, establish a mixed integer linear programming model on the basis of the running law of the train. According to the characteristics of the problem, the solution is solved by the split cutting algorithm with the optimized software. Finally the paper sets the Changchun-Siping section in main lines of Beijing-harbin as an example and verify the feasibility and availability of this method.
\end{abstract}

\section{Introduction}

In recent years, the rapid development of high speed railway has gradually affected the way of people's travel. However, there are still a variety of trains to meet the needs of different passengers on the existing busy trunk lines. Due to the complexity of China railway operation mode, the existing busy trunk lines have many kinds of trains, large density, and different equipment in each station. It is easy to interfere with the normal operation of train and even the local road network because of the interference of a certain interval, which leads to the disorder of the operation order and even the operation accident. The railway dispatching department carries out the railway traffic organization under the abnormal condition. Therefore, it is an important practical significance to study the adjustment of the interval blockade.

In case of interference, relevant research have done at domestic and abroad. Andrea D' Ariano and Francesco Corman have done a lot of research on the real-time adjustment of the train operation under the interference condition. They abstract the train operation into a non wait work shop scheduling problem with resource constraints. Solving the problem of collision route selection by using comparison chart[1]. Meng lingyun, etc propose a two stage stochastic expectation model with compensation for the uncertainty capacity failure of a single line railway and using the branch and bound method is used to solve it[2]. Wang Li , etc set up a double deck planning model for the train operation adjustment under the speed limit condition in the line interval of high speed railway, taking some measures such as stopping some trains moving to reduce the impact of emergencies on the whole process of train operation[3]. Combination with the actual railway transportation in China, there are few literatures on the real-time adjustment of the train operation after the interference of the busy trunk line interval.This paper establish a real time adjustment of mixed integer linear programming model for train operation. Setting the Changchun-Siping section in main lines of Beijing-harbin as an example and verify the feasibility and availability of this method. 


\section{The Description and Definition of the Problem}

\section{The Description and Definition of This Problem}

This paper uses the train operation diagram to solve the problem of train operation adjustment under the condition of single interval blockade. It is a complex combinatorial optimization problem. This paper combines the practice of railway dispatching. In the case of a single direction section blockade, the dispatcher will organize two-way trains running together in the section blockade. If there are still trucks at the both ends of the section, it is possible to decide whether to cancel the operation of freight train according to the real-time situation, so as to ensure the organization order of passenger trains as far as possible. If the train stops at other intermediate stations in the section, According to the principle of "first passengers and later goods, first fast and later slow", the train will be set forward. The real time adjustment is combined with the practice of scheduling operation. The optimal train operation adjustment scheme is obtained.

\section{The Hypothesis of the Problem}

(1)Each line of the busy trunk line meets the conditions for two-way traffic.

(2) Arrival-Departure Tracks in each station can meet the dual route, and they also can receive various conditions of the train.

(3)The capacity of the technical station at both ends of the fault interval is large enough.

(4) The freight trains that have not been sent from both ends of the technical station can be cancelled to ensure the passenger trains transportation organization order.

\section{Model Construction}

\section{The Abstract of the Problem}

Events, train activities and interval activities are introduced to describe the problem of train operation adjustment in the case of single direction section blockade in this paper. The event is used to indicate the train arrives at the station or set out from the station. The train activity is used to indicate the arrival of the train the train set out from a station and arrive at the next station, or arrive at a station to set out from a station. The interval activity indicate two train arrival interval, departure interval at the same station, following interval in block section.

\section{Objective Function and Basic Model}

Using $\mathrm{O}$ to indicate the set of events, $o \in O$. Using $\mathrm{L}$ to indicate the collection of all trains, $l \in L$. Using $\mathrm{S}$ to indicate the collection of all the stations, $s \in S$. For any event $o \in O$, Parameter $p_{o}$ indicate the time occurrence of event o in the planned train diagram. Similar, Parameter $q_{o}$ indicates the actual occurrence time. The decision variable $\omega_{o}$ indicates the late time of the event $o$. The parameter $\tau_{o}$ indicate the penalty value for the unit late time of event o. take $M_{1}=1440 . y_{l}$ indicates whether to cancel train III, when $y_{l}=1$, The train is cancelled. When $y_{l}=0$, Train running. The parameter $\theta_{l}$ indicates the penalty value for the train $\mathrm{L}$ to be cancelled. For every $\operatorname{train} l \in L$, The basic model without considering the capacity of each station and the interaction of trains is as follow:

$$
\operatorname{Min} \sum_{o \in O} \omega_{o} \tau_{o}+\sum_{l \in L} \theta_{l} y_{l}
$$

s.t.

$$
\begin{gathered}
0 \leq q_{o}^{I, I I}-p_{o}^{I, I I} \leq \omega_{o}^{I, I I} \quad \forall o \in O \\
M_{1} y_{l_{o}} \leq q_{o}^{I I I}-p_{o}^{I I I} \leq M_{1}, \quad l_{o} \in L^{I I I} \quad \forall o \in O_{s f}^{\text {dep }}
\end{gathered}
$$




$$
\begin{gathered}
\omega_{o}^{I I I} \geq q_{o}^{I I I}-p_{o}^{I I I}-M_{1} y_{l_{o}}, \quad l_{o} \in L^{I I I} \quad \forall o \in O \\
y_{l_{o}} \in\{0,1\} \quad l_{o} \in L^{I I I} \\
q_{o}, p_{o}, \omega_{o} \geq 0
\end{gathered}
$$

The following description of the parameters: $q_{o}^{I, I I}, p_{o}^{I, I I}, \omega_{o}^{I, I I}$ indicate train I and train II actual arrival and departure time, scheme arrival and departure time, delay time. $q_{o}^{I I I}, p_{o}^{I I I}, \omega_{o}^{I I I}$ indicate train III actual arrival and departure time, scheme arrival and departure time, delay time. $O_{s f}^{d e p}$ indicates district stations aggregate of starting trains.

Objective function formula (1) represent the minimum weighted summation of train cancellation penalty value and train late penalty value. The constraint condition (2) indicates that the actual arrival time of the train at a station can not be earlier than the scheduled arrival time. The constraint condition formula (3) indicates the cancellation of train III by moving the train line of train III to 24 hours later. The constraint condition (4) indicates the late time of train III . The constraint conditions formula (5) and formula (6) indicate the range of the value of the limited variable.

\section{Other Constraints}

When setting up the constraint conditions, it should combine the equipment conditions of the existing busy trunk lines, basic rules of railway transport organization, rules of transportation organization under abnormal operation of Railway, the rules of transport organization under the assumptions. The specific constraints are as follows:

(1)Train running time and stop time at the station constraints

In course of the operation of one train in a blockade section, each train must meet its minimum running time constraints in each section and stop time constraints at each station during the operation. Using $H_{\text {train }}$ to indicate the collection of the train activity. The specific constraints are as follows:

$$
q_{r}-q_{o} \geq J_{h} \quad \forall h=(a) \in H_{r}
$$

In formula: $\mathrm{o}, \mathrm{r}$ indicate that the same train occupies two adjacent events of the same resource successively. $h=(0, r)$ indicate 1 train activities. It can be not only the operation of the train in the interval, but also the stop of the train at the station. When $\mathrm{h}$ indicates an interval running activity, $J_{h}$ indicates the minimum running time of the train in the section. When $\mathrm{h}$ indicates that the station stops, indicates the minimum stop time of the train in the station. $J_{h}$ indicates the minimum stop time of the train in the station.

(2)Constraint of running interval of adjacent train

Under normal circumstances, the operating intervals of the existing busy trunk lines only include departure intervals, arrival interval constraint between adjacent identical trains. When the single direction interval is blocked. The fault interval within the fault period will organize two ways traffic. The reverse train enter the blockade section meet arrival and departure constraint. Here define a $0-1$ variable that indicates the order of events $\sigma_{o r}$.

$$
\sigma_{o r}= \begin{cases}1 & \text { Event } o \text { occurs before the event } r \\ 0 & \text { Otherwise }\end{cases}
$$

Between the adjacent trains in any sections in any time and reverse adjacent train in blockade section of blockade time must meet minimum running interval constraint. Indicating $H_{\text {space }}$ as interval activity aggregate. As shown in formula (9) and formula (10).

$$
q_{r}-q_{o}+M_{2}\left(1-\sigma_{o r}\right) \geq J_{h}
$$




$$
\sigma_{o r}+\sigma_{r o}=1 \quad \forall h=(o, r) \in H_{\text {space }} \wedge(r, o) \in H_{\text {space }}
$$

In formula: o, r,are two adjacent events that need to occupy the running resources of the same train. $\mathrm{h}=(\mathrm{o}, \mathrm{r})$ indicates reverse adjacent trains interval activity. Here it make $M_{2}$ large enough and far larger than $J_{h}$ to ensure that inequality is meaningful. Formula (10) indicates train interval must meet in order.

(3) The departure order restriction of train at each station in the section

When a busy trunk is blocked is fault and a single direction section is blocked, there will be a large number of train stops at the both ends of the blockade interval or even in other stations in the section where the block is located. It take the train for stop operation to determine the order of the train's departure by the priority of the train. indicating $i, j$ as the grade of the train. The constraints established are as follows:

i. For different grade of trains

$$
q_{r}^{i}-q_{o}^{j}+M_{2}\left(1-\lambda_{o r}\right) \geq J_{h}, \quad \forall h=(o, r) \in H_{\text {space }}, \quad \forall o, r \in O_{s}^{\text {dep }}, i<j, l_{o} \neq l_{r}
$$

ii. For the same grade of trains

$$
q_{r}^{i}-q_{o}^{j}+M_{2}\left(1-\lambda_{o r}\right) \geq J_{h}, \quad \forall h=(o, r) \in H_{\text {space }}, \quad \forall o, r \in O_{s}^{\text {dep }}, \quad i=j, \quad l_{o} \neq l_{r}, \quad q_{o}^{j} \leq q_{r^{\prime}}^{i}, \quad o^{\prime}, r^{\prime} \in O_{s}^{\text {arr }}
$$

(4) Trains cancel constraint

According to the characteristics of the railway operation in China, passenger trains running on the lines in the event of failure generally can't be cancelled. However, for the freight train at the time of the failure which is not setting out from the departure station. The dispatcher combine the real time running condition of the train to cancel a freight train that has not yet been sent from a technical station at both end so as to minimize the effect of fault on train operation. Combined with the actual situation, the train cancellation constraints are added as follows:

$$
\begin{gathered}
y_{l^{I, I I}}=0 \quad \forall l^{I, I I} \in L \\
y_{l^{I I I}}=0 \quad \forall l^{I I I} \in L \quad q_{s f}^{d e p} \leq U_{d i}^{s t}
\end{gathered}
$$

In formulation, $l^{I, I I}$ indicates train I or train II, $l^{I I I}$ indicates train III, $q_{s f}^{\text {dep }}$ indicates the departure time of train III at the departure station.

\section{Model Solution}

This paper built the real time adjustment model of train operation under the condition of single direction blockade of existing busy trunk lines. This article is based on the requirements of the model, we use CPLEX software to solve the model. The train in the selected section is abstracted into different classes according to the grade. Events, train activities, and interval activities are abstracted as the attributes of various types of trains. The cancellation of the train, the actual occurrence time of the train and the delay time of the train are abstracted as the decision variables. On this basis, the train plan will be read to the planned and the actual time for arrival and departure of the train. The branch cutting algorithm built in CPLEX software is used to solve the model.

\section{Example Analysis}

\section{Example Description}

This example data originate from the 2015 the Changchun-Siping section in main lines of Beijing-harbin actual operation data. Full length of Changchun-Siping section is 115KM. It contains 9 intermediate stations. There are 10 intervals in this section. The example of this article considers the operation data of the train in the section of the train diagram from 12 noon to $8 \mathrm{pm}$. 


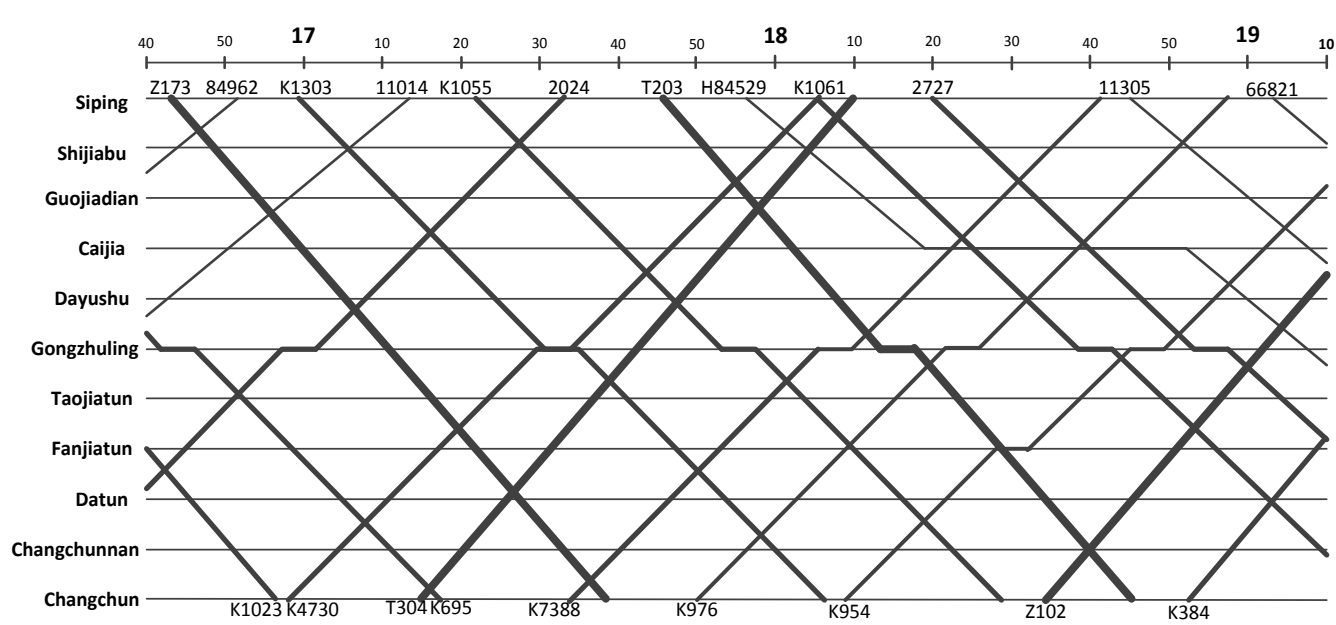

Fig.1 Train diagram under Scene 2

The penalty coefficient $\theta_{l}$ of canceling one train of train III is 500. The penalty coefficient of train I , train II for 1 [min] arrive late in every station is 10 . The penalty coefficient of train III for $1[\mathrm{~min}]$ arrive late in every station is $5 . M_{2}=10000$.

Here setting up two different scenarios to test the proposed model.

Scene 1: 13:00 the Changchun-Siping section in main lines of Beijing-harbin between Dayushu station and Caijia station downlink interval line fault, the dispatcher issued blockade downlink interval scheduling command. It is expected that the train will be restored by 13:30.

Scene 2: 17:00 the Changchun-Siping section in main lines of Beijing-harbin between Taojiatun station and Fanjiatun station downlink interval line fault, the dispatcher issued blockade downlink interval scheduling command. It is expected that the train will be restored by 18:00

Table1 Train operation adjustment results under different blockade time and blockade scene

\begin{tabular}{rcll}
\hline scene & Time of occurrence & Occurrence section & Duration \\
\hline 1 & $13: 00$ & Dayushu-Caijia & $30 \mathrm{~min}$ \\
\hline 2 & $17: 00$ & Taojiatun-Fanjiatun & $60 \mathrm{~min}$ \\
\hline
\end{tabular}

\section{Model Solution}

Based on the example of the 4.1 section and the values of the related parameters, this article uses IBM ILOG OPL programming. Running environment is Intel(R) Core (TM)i7-7500U 2.70GHz, The solution engine calls IBM ILOG CPLEX12.6.3. The CPLEX parameters use the default value, the calculation time is not more than 180s. For the example and the hypothesis scenario in the 4.1 section, the result of the solution is shown in Table 3 .

Table2 Train operation adjustment results under two scenarios

\begin{tabular}{rrrcccc}
\hline scene & $\begin{array}{l}\text { Time of } \\
\text { occurrence }\end{array}$ & $\begin{array}{l}\text { target } \\
\text { value }\end{array}$ & $\begin{array}{l}\text { Cancel the } \\
\text { train }\end{array}$ & $\begin{array}{l}\text { delay time of } \\
\text { train }\end{array}$ & Total delay time & $\begin{array}{c}\text { Total } \\
\text { calculation time }\end{array}$ \\
\hline 1 & $13: 00$ & 2250 & 0 & 5 & $293 \mathrm{~min}$ & $106 \mathrm{~s}$ \\
\hline 2 & $17: 00$ & 2700 & 0 & 4 & $274 \mathrm{~min}$ & $133 \mathrm{~s}$ \\
\hline
\end{tabular}

As can be seen from the results of the calculation in Table 3, Train operation adjustment problem with short interval blocking time, for train operation adjustment problem with short interval blocking time, this model can quickly solve the train operation adjustment scheme. Fig. 3 gives an adjustment diagram for the train operation. Rough line, the line between rough and fine, fine line in a graph indicate train I, train II, train III operation line. The dark rectangle area indicates the blocking interval and the closing and stopping time. 


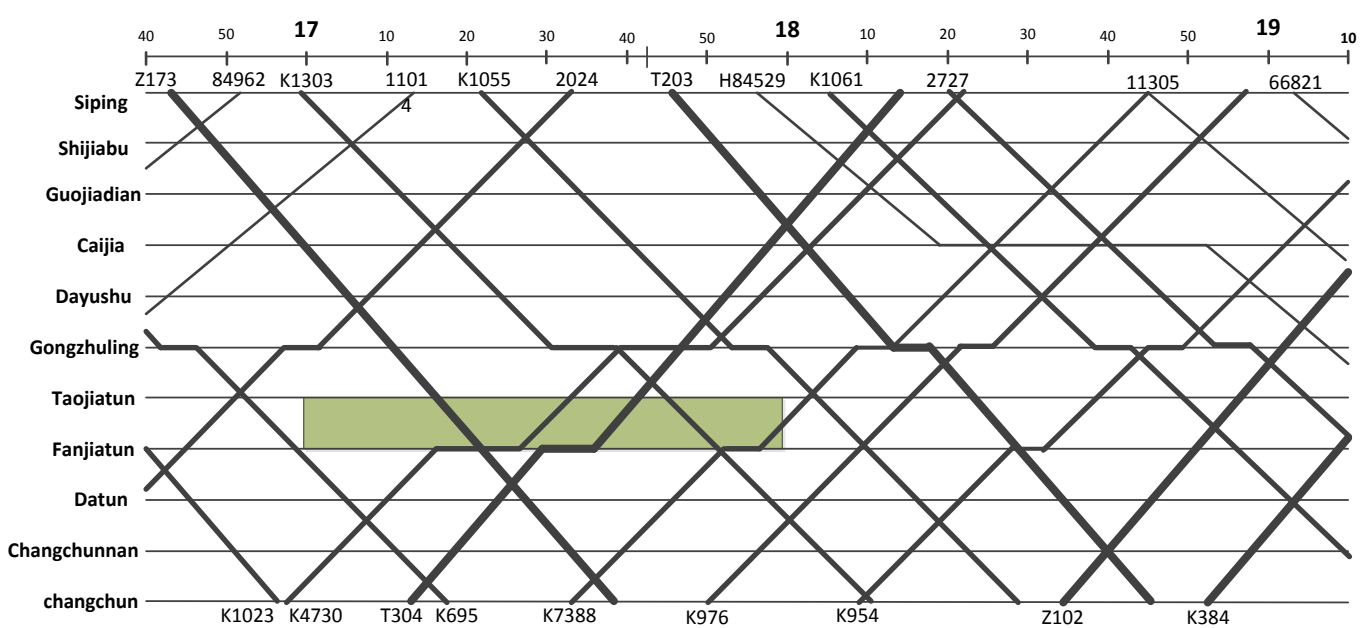

Fig.2 Adjustment diagram of train operation under scene 2

\section{Conclusion}

This paper studies the problem of train operation adjustment under the condition of busy trunk block. Establish an operation relationship between trains according to the events and activities. establish specific constraints through the characteristics of the existing busy trunk equipment and the type of train operation. We set the Changchun-Siping section in main lines of Beijing-harbin train running data at a specific time period as an example. The results show that the train operation adjustment scheme can be quickly obtained under the condition of short blockade time. It shows high practical value. But it does not consider whether is also suitable for large-scale trains or a long interval blockade. In the future research, it is necessary to use the algorithm of rolling time and so on to speed up the solution efficiency on the basis of the established model. It is convenient for the dispatchers to make decisions to improve the transportation efficiency of the existing busy trunk lines.

\section{Acknowledgements}

This work was financially supported by National Key R \& D Program during the 13th Five-Year Plan Period(2016YFB1200401).

\section{References}

[1] CORMAN F, D' Ariano A, PACCIARELli D, et al, A Tabu Search Algorithm for Rerouting trains During Rail Operations[J]. Transportation Research Part B: Methodological,2010,44 (1):175-192.

[2] MENG Lingyun, YANG Zhaoxia, LI Haiying. Train Dispatching Models under Field Capacity Breakdowns on Single-track Railway Lines[J]Systems Engineering theory \& practice, 2012,32,(4):885-894.

[3] WANG Li, QIN Yong, XU Jie, et al. Train operation in railway emergency[J]. Journal of Southeast University(Natural Science Edition), 2013, 43(1): 12-17.

[4] YANG L, ZHOU X, GAO Z. Credibility-based Rescheduling Model in a Double track Railway Network: a Fuzzy Reliable Optimization Approach[J]. Omega, 2014, 48:75-93

[5] ZHAN Shuguang, ZHAO Jun, PENG Qiyuan. Real time Train Rescheduling on High-speed Railway under Partial Segment Blockades[J]. Journal of the China railway society, 2016, 38(10):1-13. 